\title{
Effect of a bioabsorbable, super-high molecular weight poly-D,L-lactic acid plate containing recombinant human bone morphogenetic protein-2 for fracture healing
}

\author{
NING-FENG ZHOU ${ }^{1}$, YU-FENG HUANG ${ }^{1}$ and JIN-WU WANG ${ }^{2}$ \\ ${ }^{1}$ Department of Spinal Surgery, Shanghai East Hospital Affiliated to Shanghai Tongji University, Shanghai 200120; \\ ${ }^{2}$ Department of Orthopedics, Shanghai Ninth People's Hospital Affiliated to Shanghai Jiaotong University, \\ Shanghai 200011, P.R. China
}

Received December 12, 2013; Accepted July 2, 2015

DOI: $10.3892 / \mathrm{etm} .2015 .2750$

\begin{abstract}
The aim of this study was to investigate the effect of a bioabsorbable, super-high molecular weight poly-D,L-lactic acid (PDLLA) plate exhibiting the sustained release of recombinant human bone morphogenetic protein-2 (rhBMP-2) (PDLLA-rhBMP-2) on the treatment of fracture with internal fixation. A total of 32 New Zealand rabbits were randomly allocated to one of four groups (2, 4, 8 and 12 weeks), and a 2.5-mm middle ulnar osteotomy was performed bilaterally. The right side (experimental side) was fixed internally with PDLLA-rhBMP-2, and the left side (control side) was fixed with a normal PDLLA plate. At 2, 4, 8 and 12 weeks after surgery, the gross pathology of the ulnas was examined and radiographic, histological and computer image analyses were performed. The results demonstrated that the ulna fractures were fixed stably with the two bioactive plates at 2, 4, 8 and 12 weeks after surgery. At the 8-week time-point, 7 rabbits exhibited good healing at the osteotomy site on the experimental side. At 12 weeks after surgery, 8 rabbits exhibited good healing at the osteotomy site on both sides, but the experimental side showed enhanced compatibility between the plates and surrounding tissue, faster bone formation, a greater bone regeneration mass and better medullary canal structure compared with the control side. In conclusion, PPLLA-rhBMP-2 may be effectively used to treat fracture or nonunion at a non-weight-bearing site.
\end{abstract}

Correspondence to: Professor Jin-Wu Wang, Department of Orthopedics, Shanghai Ninth People's Hospital Affiliated to Shanghai Jiaotong University, 639 Zhi Zao Ju Road, Shanghai 200011, P.R. China

E-mail: wangjwtj@126.com

Dr Yu-Feng Huang, Department of Spine Surgery, Shanghai East Hospital Affiliated to Shanghai Tongji University, 150 Jimo Road, Shanghai 200120, P.R. China

E-mail: huangyufengshjt@sohu.com

Key words: bone morphogenetic protein, poly-D,L-lactic acid, fracture fixation, drug carriers, PPLLA-rhBMP-2

\section{Introduction}

Since the initial separation and naming of bone morphogenetic protein (BMP) by Urist and Strates in 1971 (1), there have been significant advances in the development of recombinant human BMP (rhBMP) (2-4). Studies have demonstrated that rhBMP-2 exhibits good osteoinductive activity, inducing the transformation of undifferentiated mesenchymal cells and osteoblasts into chondrocytes and skeletogenous cells and contributing to the formation of cartilage and bone (5-7). Since the bioactivity of rhBMP-2 is easily lost due to diffusion and degradation in the body fluid, it is necessary to develop a suitable carrier that enables the slow release of rhBMP-2 to achieve the strongest efficacy $(6,8)$.

With the development of materials science for controlling and targeting release, it is currently possible to increase the efficacy of drugs with a short half-life, such as rhBMP-2 $(2,9)$. Bioabsorbable polymerized materials, which exhibit the same strength as human compact bone and can be made into any shape, are commonly and effectively used as a carrier for BMP in order to achieve good osteoinductive activity $(2,10)$; therefore, it may be possible to develop a novel absorbable, bioactive compound plate containing rhBMP-2, which is beneficial for bone growth and fracture healing, to replace the implant currently used in clinical practice. This may be an effective way to treat fracture or nonunion at a non-weight-bearing site. At present, poly-D,L-lactic acid (PDLLA) is one of the most commonly used bioabsorbable polymerized materials for drug carriers (11). In the present study, a super-high molecular weight PDLLA plate exhibiting the sustained release of rhBMP-2 (PDLLA-rhBMP-2) was designed, and its effects on the treatment of fracture, defect recovery and fixation and degradation were evaluated, in order to provide a foundation for the future study of bone frame tissue engineering and for the clinical application of such designs.

\section{Materials and methods}

Materials. The rhBMP-2, dissolved in glycine buffer, was provided by the Huadong Gene Technology Research Center (Hangzhou, China). The rhBMP-2 had a molecular weight of 
$26 \mathrm{kDa}$, an isoelectric point of 6.56 and alkaline phosphatase activity of $>100,000 \mathrm{U} / \mathrm{mg}$. Each plate contained $0.05 \mathrm{mg}$ rhBMP-2 with super-high molecular weight PDLLA. The plate was then borated using a mold, and a porous compound surface was formed using laminating technology. Having been vacuumed, refrigerated and dried, the plate was sterilized using Co-60. The PDLLA-rhBMP-2 exhibited the following specifications (which were identical to those of the normal super-high molecular weight PDLLA plate): Length, $40 \mathrm{~mm}$; width, $9 \mathrm{~mm}$; thickness, $3 \mathrm{~mm}$; tensile strength, >50 MPa; tri-point bending strength, $>90 \mathrm{MPa}$; raw material intrinsic viscosity, $1.6 \mathrm{dl} / \mathrm{g}$; bioabsorbable internal fixing screw diameter, $2 \mathrm{~mm}$. The PDLLA-rhBMP-2 and normal super-high molecular weight PDLLA plate were made by Sichuan Dikang Sci \& Tech Pharmaceutical Industry Co., Ltd. (Chengdu, China). Other materials included an Agfa computed radiography instrument (Agfa Healthcare, Bonn, Germany), a light microscope (Leica Microsystems GmbH, Wetzlar, Germany) and a high-resolution, color image analysis system (Motic ${ }^{\circledR}$ Images Advanced 3.0; Department of Human Anatomy and Histoembryology, Shanghai Fudan University, Shanghai, China).

Animal models and grouping. A total of 32 healthy New Zealand rabbits $(3.0 \pm 0.5 \mathrm{~kg})$ were purchased from the Experimental Animal Center of the Shanghai No. 6 Municipal People's Hospital (Shanghai, China). Ketamine (0.1 g/rabbit) and $2.5 \%$ sodium pentobarbital $(30 \mathrm{mg} / \mathrm{kg})$ were used to anesthetize the rabbits. Following disinfection and the placement of surgical towels, the dorsal aspect of the ulnar was cut with a straight incision. The extensors and flexors of the forearm were then bluntly dissected, and parts of the periosteum were cut open and removed to expose the ulnar stem. The middle of the ulna was cut with a scroll saw, and the broken ends were planished with a dentistry bodkin, resulting in a $2.5-\mathrm{mm}$ defect in the periosteum of the ulna. The right side, which was used as the experimental side, was fixed internally with the PDLLA-rhBMP-2 and the left side, which was the control side, was fixed with the normal PDLLA plate. Each broken end of the fractured bone was fixed using two bioabsorbable screws. Finally, the incision was rinsed with gentamicin sulfate and sutured layer-by-layer. The animals were placed back in the cage and fed with normal forage. Every other day, 80,000 units gentamicin sulfate was hypodermically injected for three times in total. The animals were examined at 2, 4, 8 and 12 weeks after surgery.

Radiographic examination. The animals were anesthetized and an AP projection of the ulna and radius was captured with the following conditions: $40 \mathrm{KV}, 50 \mathrm{~mA}, 0.2 \mathrm{sec}$ and $60-\mathrm{cm}$ distance. The callus density, which was the density of the identical area of bone defect on the same graph was 100, and the identical area of ground color on the graph was 0 . The relative magnitude of the callus density of the region was measured as the bone defect. The averaged callus density of the bone defect represented the mean of the visible region.

Visual analysis. At 2, 4,8 and 12 weeks after surgery, 8 animals were sacrificed by i.v. pentobarbital sodium $(60 \mathrm{mg} / \mathrm{kg})$, respectively, and the ulna and radius in the region of the bone defect were cut. The degradation of the implant, compatibility between the bone and implant, reaction of the surrounding soft tissue, material degradation and fragmentation, ossification and inflammatory reactions were assessed using the methods described below.

Histopathological analysis. The broken ends of the fractured ulna (PDLLA-rhBMP-2 or control PDLLA plate) were isolated, fixed using $10 \%$ formaldehyde solution, decalcified, embedded in paraffin and cut into 5- $\mu \mathrm{m}$ laterigrade and longitudinal serial sections. The sections were subsequently stained using hematoxylin and eosin and observed under an optical microscope. Images were captured for the analysis of the percentage of the target area (newly formed bone region).

Statistical analysis. Data are presented as the mean \pm standard deviation, and one-way analysis of variance was performed using the SPSS 13.0 statistical software (SPSS, Inc., Chicago, IL, USA). $\mathrm{P}<0.05$ was considered to indicate a statistically significant difference.

\section{Results}

Radiographic examination. The results of the radiographic examination showed that the ulna fractures were fixed stably with the two bioactive plates. No movement was observed, and good reduction was maintained. At 2 weeks after surgery, the experimental sides, which contained the PDLLA-rhBMP-2, exhibited some callus formation, but the fracture line remained evident; the control sides with the normal PDLLA plate had no visible callus formation (Fig. 1A). At 4 weeks after surgery, the experimental sides with the PDLLA-rhBMP-2 exhibited extensive lamellar callus formation, and the fracture line was ambiguous; however, the control sides with the normal PDLLA plate showed only limited osteotylus formation, with a thinner callus and a visible fracture line (Fig. 1B). At 8 weeks after surgery, the experimental sides with the PDLLA-rhBMP-2 exhibited synostosis of the ulna and the disappearance of the fracture line; by contrast, the control sides with the normal PDLLA plate exhibited crumbly callus formation, and the fracture line was cloudy and blurred (Fig. 1C). At 12 weeks after surgery, the experimental sides with the PDLLA-rhBMP-2 exhibited complete synostosis of the ulna and a mounded callus, while the control sides with the normal PDLLA plate exhibited extensive callus formation and the disappearance of fracture line (Fig. 1D). The callus density of fracture recovery for each side at different phases is shown in Table I.

Visual analysis. At 1 week after surgery, the animals in each group exhibited primary healing, and no red swelling of the skin, effusion, wound dehiscence or histological material discharge was observed. The two bioactive plates fixed stably, and the quantity of new bone increased as time passed; in addition, the materials degraded gradually. At 8 weeks after surgery, the experimental side in 7 animals had recovered from the fracture, and the control side in 2 animals had some callus formation. The bone defect in the other sides was mainly filled with fibrous connective tissue. At 12 weeks after surgery, all animals showed defect recovery, irrespective of the type of plate, and part of the surface of both types of plate had begun to be degraded and absorbed; however, the formation of new 
Table I. Results of the radiographic examination.

\begin{tabular}{|c|c|c|c|c|c|c|}
\hline \multirow[b]{2}{*}{ Group } & \multicolumn{4}{|c|}{ Post-operative time (weeks) } & \multirow[b]{2}{*}{ F-value } & \multirow[b]{2}{*}{ P-value } \\
\hline & 2 & 4 & 8 & 12 & & \\
\hline Experimental & $39.22 \pm 2.48$ & $48.79 \pm 1.26$ & $63.78 \pm 1.78$ & $78.60 \pm 1.25$ & 10.963 & 0.003 \\
\hline Control & $33.83 \pm 1.13$ & $41.28 \pm 1.25$ & $55.23 \pm 0.68$ & $66.54 \pm 1.33$ & 17.602 & 0.001 \\
\hline $\mathrm{t}$ & 5.031 & 8.005 & 10.110 & 0.980 & & \\
\hline P-value & 0.007 & 0.001 & 0.001 & 0.038 & & \\
\hline
\end{tabular}
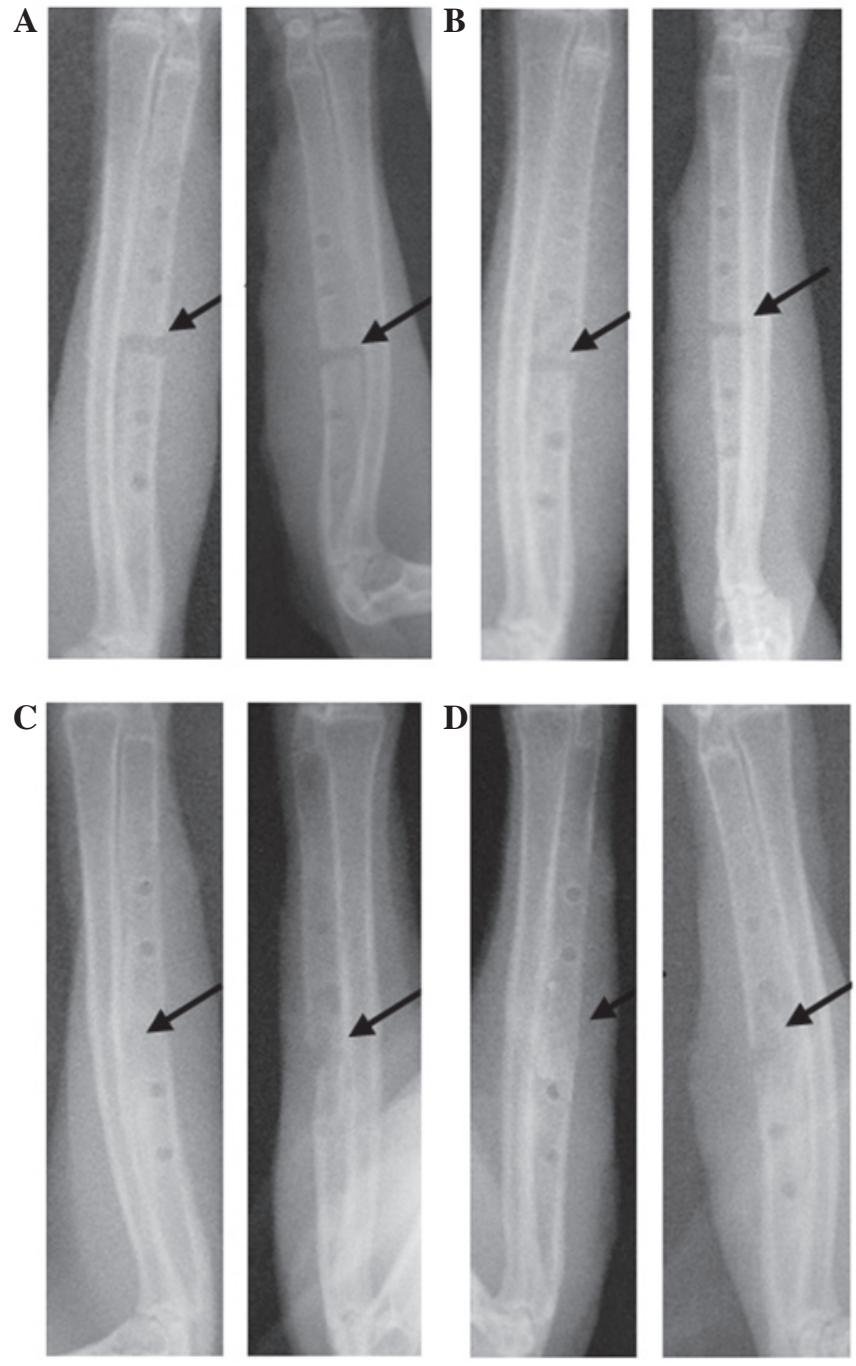

Figure 1. Results of the radiographic examination at (A) 2, (B) 4, (C) 8 and (D) 12 weeks after surgery. The left panel in parts (A-D) represents the experimental side, and the right panel represents the control side.

bone and the degradation of the plates in the experimental side were notably faster. All the plates were far from completely absorbed.

Histopathological examination. At 2 weeks after surgery, no obvious inflammatory reaction was observed on either side; the experimental sides had a small quantity of new bone, ground substance and cells, which expanded across the surface between the bone and the materials. Furthermore, blood capil- laries appeared in the interface near the soft tissue (Fig. 2). At 4 weeks post-surgery, no obvious degradation or inflammatory reaction was found on either side. The control sides had a small quantity of new bone, ground substance and cells, which expanded across the surface between the bone and the materials, and numerous blood capillaries appeared in the interface near the soft tissue; however, the experimental sides exhibited the formation of new bone trabeculae, blood capillaries and fibroblasts that almost filled the defect between the interface of the bone and the implanted materials. Furthermore, the initially formed cavitas medullaris and the broken ends of the fractured bone were linked well by a fibrous callus (Fig. 2). At 8 weeks after surgery, the plates in both sides had very little degradation and absorption and showed good biocompatibility, with no reaction of inflammatory histocytes. The control sides with the normal PDLLA plate had a large quantity of new bone, ground substance and cells, which spread across the surface between the bone and the implanted materials; blood capillaries appeared in the interface near the soft tissue; woven bone formation was observed; and the broken ends of the fractured bone were linked by a fibrous callus. The experimental sides with the PDLLA-rhBMP-2 exhibited a larger area of newly formed bone, dynamic rebuilding of the bone tissue and neogenesis, numerous osteoblasts and blood capillaries around the new bone tissue, mature bone tissue, fewer cellular components and calcified nodules in certain regions (Fig. 2). At 12 weeks after surgery, the plates in both sides had partly undergone degradation and absorption. In the control sides with the normal PDLLA plates, numerous osteoblasts and blood capillaries were observed around the new bone tissue and woven bone. Furthermore, the appearance of lamellar bone, reduced ingredient of cells and calcified nodules in certain regions were observed. In the experimental sides, which contained the PDLLA-rhBMP-2, there were numerous mature bone tissues and lamellar bone. In addition, the plate absorption was quicker than that in the control sides, showing enhanced biocompatibility and bone healing, and there was a partial appearance of the cavitas medullaris (Fig. 2).

Computer image analysis. The percentage area of newly formed bone at the same side of bone defect was analyzed with image analysis software (show in Table II). At 2, 4 and 8 weeks after surgery, a significant difference was found in the area of newly formed bone between the experimental and control sides $(\mathrm{P}<0.01$ for 2 and 4 weeks, $\mathrm{P}<0.05$ for 8 weeks). It was evident that the formation of new bone in the experimental side was more rapid than that in the control side. At 12 weeks after surgery, 
Table II. Percentage area of newly formed bone at the same side of the bone defect analyzed using image analysis software.

\begin{tabular}{|c|c|c|c|c|c|c|}
\hline \multirow[b]{2}{*}{ Group } & \multicolumn{4}{|c|}{ Post-operative time (weeks) } & \multirow[b]{2}{*}{ F-value } & \multirow[b]{2}{*}{ P-value } \\
\hline & 2 & 4 & 8 & 12 & & \\
\hline Experimental & $0.106 \pm 0.015$ & $0.292 \pm 0.019$ & $0.457 \pm 0.048$ & $0.574 \pm 0.047$ & 130.285 & $<0.001$ \\
\hline Control & 0 & $0.193 \pm 0.019$ & $0.339 \pm 0.029$ & $0.601 \pm 0.037$ & 204.855 & $<0.001$ \\
\hline $\mathrm{t}$ & 17.909 & 6.525 & 3.672 & 0.778 & & \\
\hline P-value & 0.003 & 0.003 & 0.021 & 0.480 & & \\
\hline
\end{tabular}

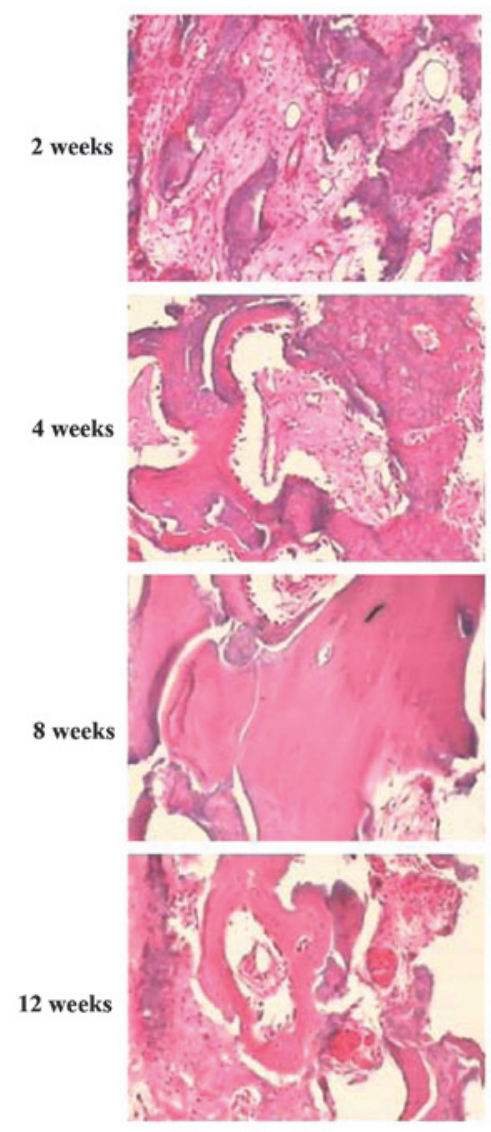

Experimental group
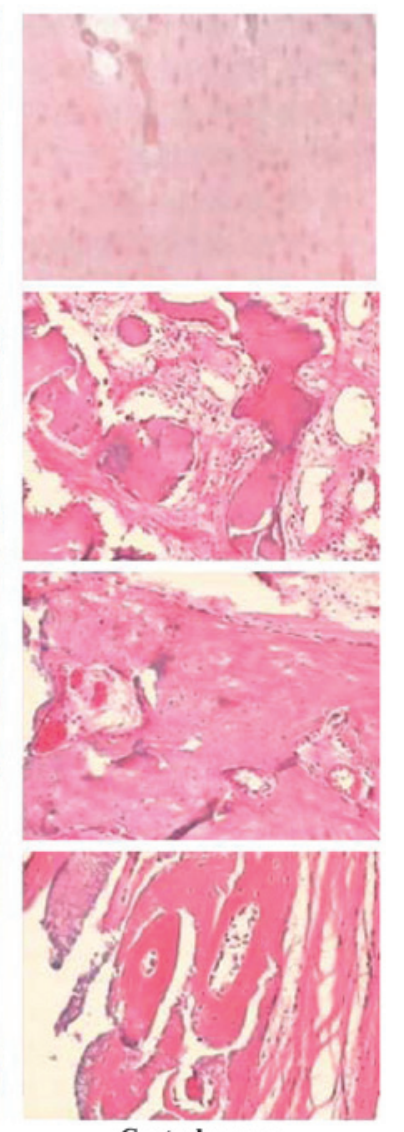

Control group
Figure 2. Results of the histological analysis. The left panels represent the experimental side, and the right panels represent the control side.

no significant difference in the area of newly formed bone was found between the experimental and control sides $(\mathrm{P}>0.05)$.

\section{Discussion}

In in vitro culture, rhBMP-2 facilitates the differentiation of progenitor cells into osteoblasts, but has a depressive effect on the differentiation of muscle plasma cells (12). Previous studies have demonstrated that rhBMP-2 can stimulate the differentiation of cells derived from bone tissue, such as precursor cells of osteoblasts, W-20-17 marrow stromal cells, as well as cell lines derived from non-bone tissue, such as multipotent fibroblasts and myoblasts, to osteoblasts $(12,13)$.

rhBMP-2 can suppress the expression of genes for myoblast and myotube formation, which explains the ability of rhBMP-2 to promote the directional differentiation of precursor cells into osteoblasts $(14,15)$. In the present study, radiographic, pathohistological and computer image analyses were performed, which demonstrated that, during the first 2-8 weeks after surgery, the broken ends of the fractured bone fixed with the PDLLA-rhBMP-2 grew more rapidly than those of the control side. Since rhBMP-2 is a type of hydrophobic glycoprotein, it is stable in a acidic, hypothermal and desiccative environment $(6,8,13)$; therefore, in the present study, the hydrophobicity of the polymer was used to embed the rhBMP-2 and to isolate it from the body fluid, resulting in delayed rhBMP-2 release at a constant concentration and time. This was demonstrated to be an effective method of maintaining its osteoinductive activity.

The osteoinductive activity of BMP is generally accepted; however, its clinical use is not satisfactory. In addition to the purification and the surgical procedure, the preparation of the carrier is an important factor affecting the efficacy of BMP. Using simple BMP to produce a marked effect is challenging, as it dissolves in the body fluid; however, the use of a slow-release carrier can enhance the effect of the BMP (16). The current slow-release carriers that are commonly used include mineral salts (such as hydroxyapatite, ceramic and gypsum), biological materials (such as collagen and fibrin) and bioabsorbable polymerized materials; however, there are disadvantages associated with the majority of these carriers: Ceramic is fragile and easily smashed, hydroxyapatite cannot be degraded, gypsum produces heat when reacting with foreign materials and biological materials have immunity-related problems, which cannot be completely overcome. Bioabsorbable polymerized materials can, on the whole, solve the above problems, as their slow-release rate can be adjusted, making it possible to adjust the rates of degradation and release of BMP; therefore, bioabsorbable polymerized materials are one of the most suitable carriers $(2,17,18)$.

We have studied the bioabsorbable polymerized materials polyglycolic acid (PGA) and polylactic acid (PLA). Due to the fact that PLA maintains its strength for longer and is associated with lower degradation and tissue reaction rates and response strength than PGA, PLA has become the focus of the study of the use of bioabsorbable materials in internal fixation. PLA contains self-enhanced poly-L-lactic acid (SR-PLLA), poly-L-lactic acid (PLLA) and PDLLA and can therefore be completely absorbed by bone tissue. The degradation of PLA relies on water, and it is transformed to carbon dioxide and water by the citric acid cycle, prior to being expelled out of the body through respiration (19). 
Bergsmaju et al (20) found that PLA had not been completely absorbed in the body after 5-7 years and caused a tardive tissue reaction, which may have been due to the higher crystallinity of SR-PLLA and PLLA. PDLLA is an amorphous material and can be completely absorbed by the body in between 24 weeks and 18 months due to its good histocompatibility, biodynamic performance and adequate absorption rate; therefore, PDLLA is considered to be the most suitable and effective candidate material for use in bone technology $(11,21)$. The present study showed that it is possible to control the release rate of rhBMP-2 by controlling the aperture size and porosity of the synthesized materials to adjust the material strength and degradation time. The PDLLA-rhBMP-2 had good incipient mechanical fixation strength and slow degradation, with the constant formation of new bone, and avoided the stress-shielding effect, which can affect the healing of the fracture. In addition, the PDLLA-rhBMP-2 exhibited an enhanced fracture-healing ability, improved compatibility with the surrounding tissue, faster bone formation, an increased bone regeneration mass and enhanced medullary canal structure compared with the normal PDLLA plate.

Traditional materials for internal and external fixation are metal. These metal materials have certain disadvantages (5): i) Electrolytic tarnishing in the body; ii) higher rigidity and lower elasticity creating a stress that can protect the broken ends of fractured bone and affect the union of a fracture; iii) high weight, which can affect functional exercise and activity; iv) interference with magnetic resonance imaging and v) requirement of further surgery to remove the implant, increasing the burden and pain of the patients. Previous investigations have demonstrated that bioabsorbable polymerized materials can be used to prepare screws and fixation sticks for treating fractures $(22,23)$; however, the strength and osteoinductive activity of these materials are insufficient and the cost is high, which prevents the generalized application of these materials. In the present study, PDLLA-rhBMP-2, which exhibited good bending and tensile strength, and no conspicuous absorption and looseness, was designed. The material showed good biocompatibility and osteoinductive activity, making it an ideal fixation material for fracture repair. This plate has no requirement for further surgery to be removed, and it can enhance fracture healing; therefore, PDLLA-rhBMP-2 shows potential for treating fracture or nonunion at non-weight-bearing sites, such as the acetabulum pelvis, skull and jaw facial bones. Further research is required prior to its clinical application.

\section{Acknowledgements}

This study was supported by the Science and Technology Project of Shanghai Pudong New Area Committee (no. pkj2012-y10).

\section{References}

1. Urist MR and Strates BS: Bone morphogenetic protein. J Dent Res 50: 1392-1406, 1971

2. Whang K, Goldstick TK and Healy KE: A biodegradable polymer scaffold for delivery of osteotropic factors. Biomaterials 21: 2545-2551, 2000.
3. Ohgushi H, Goldberg VM and Caplan AI. Heterotopic osteogenesis in porous ceramics induced by marrow cells. J Orthop Res 7: 568-578. 1989.

4. Bai XD, Li G, Zhao C, Duan H and Qu F: BMP7 induces the differentiation of bone marrow-derived mesenchymal cells into chondrocytes. Med Biol Eng Comput 49: 687-692, 2011.

5. Na YH, He Y, Shuai X, Kikkawa Y, Doi Y and Inoue Y: Compatibilization effect of poly(epsilon-caprolactone)-b-poly (ethylene glycol) block copolymers and phase morphology analysis in immiscible poly(lactide)/poly(epsilon-caprolactone) blends. Biomacromolecules 3: 1179-1186, 2002.

6. Qian G, Dong YH, Yang WC and Wang M: Injectable calcium phosphate cement and fibrin sealant recombined human bone morphogenetic protein-2 composite in vertebroplasty: An animal study. Bosn J Basic Med Sci 12: 231-235, 2012.

7. Bessho K: Ectopic osteoinductive difference between purified bovine and recombinant human bone morphogenetic protein. In: Bone Morphogenetic Proteins: Biology, Biochemistry and Reconstructive Surgery. Lindholm TS (ed.). Academic Press, Ney York, NY, pp.105-111, 1996.

8. Guan Y, Wang Q, Cheng Y, Teng W and Huang H: Study on gene transfection in bone marrow mesenchymal stem cells mediated by plasmid of bone morphogenetic protein 2 loaded lipopolysaccharide-amine nanopolymersomes. Zhongguo Xiu Fu Chong Jian Wai Ke Za Zhi 28: 1292-1297, 2014 (In Chinese).

9. Langer R: New methods of drug delivery. Science 249: 1527-1533, 1990.

10. Ma J, Cao H, Li Y and Li Y: Synthesis and characterization of poly(DL-lactide)-grafted gelatins as bioabsorbable amphiphilic polymers. J Biomater Sci Polym Ed 13: 67-80, 2002.

11. Ma Z, Chen F, Zhu YJ, Cui T and Liu XY: Amorphous calcium phosphate/poly(D,L-lactic acid) composite nanofibers: Electrospinning preparation and biomineralization. J Colloid Interface Sci 359: 371-379, 2011.

12. Vukicevic S and Sampath KT (eds.): Bone Morphogenetic Proteins: From Laboratory to Clinical Practice. Birkhäuser Verlag, Berlin, pp.60-126, 2002.

13. Cirano FR, Togashi AY, Marques MM, Pustiglioni FE and Lima LA: Role of rhBMP-2 and rhBMP-7 in the metabolism and differentiation of osteoblast-like cells cultured on chemically modified titanium surfaces. J Oral Implantol 40: 655-659, 2014

14. Luangphakdy V, Shinohara K, Pan H, Boehm C, Samaranska A and Muschler GF: Evaluation of rhBMP-2/collagen/TCP-HA bone graft with and without bone marrow cells in the canine femoral multi defect model. Eur Cell Mater 29: 57-69, 2015.

15. Kandziora F, Pflugmacher R, Scholz M, Knispel C, Hiller T, Schollmeier G, Bail H, Schmidmaier G, Duda G, Raschke M and Haas NP: Comparison of BMP-2 and combined IGF-I/TGF-ss1 application in a sheep cervical spine fusion model. Eur Spine J 11: 482-493, 2002.

16. Schmidmaier G, Wildemann B, Cromme F, Kandziora F, Haas NP and Raschke M: Bone morphogenetic protein-2 coating of titanium implants increases biomechanical strength and accelerates bone remodeling in fracture treatment: A biomechanical and histological study in rats. Bone 30: 816-822, 2002.

17. Kandziora F, Scholz M, Pflugmacher R, Krummrey G, Schollmeier G, Schmidmaier G, Schnake KJ, Duda G, Raschke M and Haas NP: Experimental fusion of the sheep cervical spine. Part II: Effect of growth factors and carrier systems on interbody fusion. Chirurg 73: 1025-1038, 2002 (In German).

18. Illi OE and Feldmann CP: Stimulation of fracture healing by local application of humoral factors integrated in biodegradable implants. Eur J Pediatr Surg 8: 251-255, 1998.

19. Böstman OM: Absorbable implants for the fixation of fractures. J Bone Joint Surg Am 73: 148-153, 1991.

20. Bergsmaju E, Bruijn W, Roxema FR, Bos RR and Boering G: Late degradation tissue response to poly(L-lactide) bone plate and screws. Biomaterials 16: 25-31, 1995.

21. Suuronen R, Pohjonen T, Hietanen J and Lindqvist C: A 5-year in vitro and vivo study of biodegradation of polylactide plates. J Oral Maxillofac Surg 56: 604-614, 1998.

22. Ashammakhi N, Mäkelä EA, Vihtonen K, Rokkanen P, Kuisma H and Törmälä P: Strength retention of self-reinforced polyglycolide membrane: An experimental study. Biomaterials 16: 135-138, 1995.

23. Manninen MJ, Päivärinta U, Taurio R, Törmälä P, Suuronen R, Räihä J, Rokkanen P and Pätiälä H: Polylactide screws in the fixation of olecranon osteotomies. A mechanical study in sheep. Acta Orthop Scand 63: 437-442, 1992. 